\title{
Identification of a novel prostate cancer biomarker, caveolin-I: Implications and potential clinical benefit
}

This article was published in the following Dove Press journal:

Cancer Management and Research

7 May 2010

Number of times this article has been viewed

\author{
Paul G Corn \\ Timothy C Thompson \\ Department of Genitourinary \\ Medical Oncology, The University \\ of Texas MD Anderson Cancer \\ Center, Houston, TX, USA
}

\begin{abstract}
While prostate cancer is a common disease in men, it is uncommonly life-threatening. To better understand this phenomenon, tumor biologists have sought to elucidate the mechanisms that contribute to the development of virulent prostate cancer. The recent discovery that caveolin-1 (Cav-1) functions as an important oncogene involved in prostate cancer progression reflects the success of this effort. Cav-1 is a major structural coat protein of caveolae, specialized plasma membrane invaginations involved in multiple cellular functions, including molecular transport, cell adhesion, and signal transduction. Cav-1 is aberrantly overexpressed in human prostate cancer, with higher levels evident in metastatic versus primary sites. Intracellular Cav-1 promotes cell survival through activation of Akt and enhancement of additional growth factor pro-survival pathways. Cav-1 is also secreted as a biologically active molecule that promotes cell survival and angiogenesis within the tumor microenvironment. Secreted Cav-1 can be reproducibly detected in peripheral blood using a sensitive and specific immunoassay. Cav-1 levels distinguish men with prostate cancer from normal controls, and preoperative Cav-1 levels predict which patients are at highest risk for relapse following radical prostatectomy for localized disease. Thus, secreted Cav-1 is a promising biomarker in identifying clinically significant prostate cancer.
\end{abstract}

Keywords: caveolin-1, prostate cancer, biomarker

\section{Introduction}

Over the past decade, advances in basic tumor biology have influenced the approach to treating patients with prostate cancer. While research efforts have historically focused on the prostate cancer epithelial cell, there is growing evidence that interactions between the host tissue microenvironment and the cancer epithelial cell are critical for tumorigenesis. ${ }^{1}$ Understanding the bidirectional cancer cell-host interaction now dominates prostate cancer research. For example, prostate cancer epithelial cells preferentially metastasize to bone by acquiring osteomimetic properties that usurp normal bone homeostasis. ${ }^{2}$ Once in the bone, prostate cancer epithelial cells secrete soluble factors that act through both paracrine and autocrine mechanisms to promote cell survival and angiogenesis. This knowledge has led to novel treatment strategies that target the bone microenvironment (eg, with antiangiogenesis inhibitors) in addition to the epithelial cell (eg, with chemotherapy).

Despite this progress, however, novel biomarkers are needed to improve the ability to detect prostate cancer, predict prostate cancer lethality, and monitor response to therapy. Prostate-specific antigen (PSA) is the most widely used biomarker in prostate cancer management, but after two decades its value and appropriate use
Correspondence:Timothy Thompson Department of Genitourinary Medical Oncology, MD Anderson Cancer Center, Box 0018-3, 1515 Holcombe Boulevard, Houston, TX 77030, USA

Tel +l 7137929955

Fax +I 7137929956

Email timthomp@mdanderson.org 
remain controversial. ${ }^{3,4}$ This has led to the development of additional biomarkers to compensate for, or improve upon, the limitations of PSA. Candidate prostate cancer biomarkers now broadly include circulating tumor cells, tumor-specific genetic changes (mutations, amplifications, deletions, translocations), epigenetic changes (DNA methylation, histone modifications, microRNAs), and protein changes (increased expression of oncogenic proteins, diminished expression of tumor suppressor proteins, production of soluble tumor-associated proteins). ${ }^{5-7}$ High-throughput technologies are also significantly impacting prostate cancer biomarker research, with the potential to provide comprehensive tumor "transcriptomes" (complete set of mRNAs expressed) and "proteomes" (complete set of proteins expressed). ${ }^{8}$

To date, however, few of these markers have made the transition from research to clinical practice. Most biomarkers are attractive in theory but fail to meet the required standards for a clinically useful test with respect to sensitivity/specificity, positive/negative predictive value, reproducibility, reliability, precision, accuracy, and validity. For these reasons, the development of a successful biomarker is as challenging as the development of a successful new drug, since both discovery platforms involve screening hundreds of candidates, discarding most, and validating only a select few. ${ }^{9}$ Given the reality of limited funding for translational research, limited patient tissue resources (blood, urine, biopsy specimens, etc), and limited time to meet critical patient needs, it is essential to prioritize development of the most promising biomarkers. ${ }^{10}$

For a number or years, our laboratory has been investigating the use of caveolin-1 (Cav-1) as a novel predictive biomarker for prostate cancer progression. ${ }^{11} \mathrm{Cav}-1$ is an important regulatory molecule involved in molecular transport and cell signaling. ${ }^{12}$ While normal prostate epithelia express minimal levels of Cav-1, prostate cancer cells abundantly overexpress Cav-1, both at the tissue level and as a secreted protein in sera that can be measured by enzyme-linked immunosorbent assay (ELISA). ${ }^{13}$ In contrast with PSA, detailed mechanistic studies have shown that Cav-1 is linked to malignant characteristics of prostate cancer cells and alters the tissue microenvironment in a manner that promotes angiogenesis. ${ }^{14}$ In this review, we discuss current concepts illustrating the need for novel biomarkers in prostate cancer and the potential for Cav-1 to fill this need.

\section{Clinical features of prostate cancer}

Prostate cancer is a major health care problem in the US. It is the most common noncutaneous malignancy and the second leading cause of cancer death in men. In 2009, it is estimated that 192,280 men will be newly diagnosed with prostate cancer and 27,360 men will die from the disease. ${ }^{15}$ The greatest risk factor for developing and dying from prostate cancer is increasing age. ${ }^{16,17}$ Thus, as life expectancies increase and death rates decrease, the burden of prostate cancer on patients, families, and society continues to grow. ${ }^{18}$ Other prostate cancer risk factors include race (African-American), family history, obesity, and hereditary susceptibility loci, including BRAC1/BRACA2. ${ }^{19-22}$

Prostate cancer is a biologically and clinically heterogeneous disease. While most men who live long enough eventually develop prostate cancer, only a minority will die from it. This is evidenced by a high incidence of occult malignancy in autopsy series of men who die from non-prostate cancer causes and in clinically normal prostates of men undergoing cystoprostatectomy for bladder cancer. ${ }^{23,24}$ These data illustrate two unique features of prostate cancer that distinguish it from other solid tumor types. First, untreated prostate cancer has a relatively prolonged natural history and is often indolent for 15 years or more before becoming life-threatening. . $^{2,26}$ Second, longitudinal studies suggest that prostate cancer has the potential to be either clinically "significant" or clinically "insignificant" based on the likelihood that the cancer will threaten a person over the course of his natural lifetime. ${ }^{27}$ For these reasons, it is critical to consider a patient's age and comorbidities at the time of diagnosis, since the risk of morbidity and mortality from non-prostate cancer illnesses also increases with age and may even exceed those from prostate cancer in older patients. ${ }^{25}$

For those patients with clinically significant disease, tumor progression occurs in a well-recognized anatomic pattern. ${ }^{28}$ Tumors that are initially confined within the prostate gland first spread to locoregional lymph nodes (though not usually with radiographic enlargement) and then subsequently disseminate to distant organs, with a striking predilection for the skeleton. ${ }^{2}$ The discovery and application of hormone ablative therapy to patients with metastatic disease reveals that most tumors ( $>90 \%$ ) are initially responsive to castration but remain incurable because of the subsequent evolution of castrate-resistant disease. Thus, the greatest opportunity to cure patients with prostate cancer is through local therapy (surgery or radiation) for early-stage, small-volume, androgen-dependent, organ-confined disease. Fortunately, only a minority of patients ultimately develop metastatic castrate-resistant disease. To conceptualize the clinical heterogeneity evident during prostate cancer progression, 
patients are assigned to different "clinical disease states" to help structure treatment recommendations as well as the development of novel therapies and biomarkers. ${ }^{29,30}$

Prior to the discovery of PSA, most patients with prostate cancer were not diagnosed until they presented with clinical symptoms of advanced disease, such as bladder outlet obstruction or bony pain. These patients were rarely cured with available therapies. Following the widespread implementation of PSA screening in 1988, however, there has been a dramatic increase in the number of asymptomatic younger men detected with earlier-stage, localized disease. ${ }^{31}$ This "stage-migration" phenomenon has been accompanied by a significant increase in the number of patients undergoing radical prostatectomy. ${ }^{32}$

The advent of PSA also influenced the development of a logical strategy to reduce morbidity and mortality from prostate cancer, by detecting clinically significant cancers at their earliest stage when they are androgen-dependent and organ-confined, because these tumors have the greatest chance of being cured with local therapy (surgery or radiation). ${ }^{33,34}$ In support of this strategy, treatment of early-stage tumors with radical prostatectomy has been shown to improve survival when compared with watchful waiting in a randomized clinical trial. ${ }^{35}$ In this study, the majority of tumors were detected by digital rectal examination rather than PSA. Since tumors detected by PSA are generally of lower clinical stage than tumors detected by digital rectal examination (for example, comparing T1c versus T2 disease), one would predict that treatment of PSA-detected tumors would improve outcomes for men diagnosed with prostate cancer.

However, whether the practice of PSA screening and early therapeutic intervention has contributed to the decline in death rates from prostate cancer remains controversial. The recent publication of two large randomized screening trials (with greater than 250,000 patients) has not helped clarify the issue, given that one trial did not show a survival benefit (the Prostate, Lung, Colorectal and Ovarian Cancer Screening Trial [PLCO] in the US) while the other one did (the European Randomised Study of Screening for Prostate Cancer [ERSPC] trial in Europe). ${ }^{36,37}$ In the positive ERSPC trial, it was estimated that 1,410 men would need to be screened and 48 treated to prevent one prostate cancer death during a 10year period. Thus, the benefits of screening come at a high cost with respect to the large number of patients that need to be screened, biopsied, and treated to prevent each death.

The success of the early diagnosis-early intervention strategy has arguably been hampered by the lack of accurate clinicopathologic, prognostic, and predictive biomarkers to distinguish reliably between clinically significant and insignificant disease. For example, because most early-stage cancers are statistically likely to be clinically insignificant, many patients are "overdiagnosed" and "overtreated" because they receive local therapy for tumors that would never have harmed them. Since local therapies carry some risk for morbidity (eg, incontinence, loss of potency), this has important implications for the patient who receives unnecessary treatment. Overtreatment, in conjunction with lead-time bias due to PSA screening (where survival appears longer because of diagnosis before the cancer is clinically evident), certainly dilute the survival benefit of treating early-stage disease. ${ }^{38}$

Conversely, some patients are "undertreated" because local therapies are not uniformly curative for clinically significant disease. Reasons for this include deficiencies in execution of the therapeutic modality (for example, inadequate tumor resection by an inexperienced surgeon), localized tumors with an aggressive biology that is not altered by local therapy, and undetectable micrometastases at the time of treatment. These "undertreated" patients also diminish the potential positive impact of local treatment on survival. As evidence for this, up to $35 \%$ of patients will experience a PSA relapse following local therapy. ${ }^{39}$ These patients also suggest that efforts to improve local control and eradicate micrometastases (for example, by incorporating neoadjuvant or adjuvant therapy into local therapy) would benefit long-term patient outcomes. ${ }^{39}$ Emerging data support this hypothesis, including the use of adjuvant radiation following radical prostatectomy for high-risk, node-negative tumors, immediate treatment with hormone ablation following radical prostatectomy and lymphadenectomy for node-positive disease, and the use of adjuvant hormone ablation following radiation therapy for high-risk disease..$^{40-42}$

As the incidence of men diagnosed and treated for localized prostate cancer increases, so does the incidence of men presenting with PSA-only recurrent disease (in other words, no radiographic evidence for metastases). This scenario poses a therapeutic dilemma for physicians and considerable anxiety for patients. Remarkably, as experience with this disease state matures, it is becoming clear that PSA-only recurrences do not uniformly portend morbidity/mortality from the disease. For example, data from a large series of patients who underwent radical prostatectomy for localized disease showed that only approximately one-third of patients who experience a PSA relapse go on to develop metastases within 15 years and, for those who did, the median time to 
death was five years. ${ }^{43}$ These patients are generally offered hormone ablative therapy, commonly using an intermittent strategy. ${ }^{44}$

For patients with metastatic disease, the initial standard treatment approach is hormone ablative therapy for androgen-dependent disease. ${ }^{45}$ However, this therapy is not curative and most patients eventually develop castrate-resistant progression within 12 to 18 months. ${ }^{46}$ Castrate-resistant prostate cancer is defined as progression when the serum testosterone level is $<50 \mathrm{ng} / \mathrm{dL} .{ }^{30}$ Even in the clinically castrate disease state, however, there is growing evidence that prostate cancer cells continue to rely on androgen receptor signaling by utilizing extragonadal sources of testosterone produced in the adrenal gland and by the tumor itself. ${ }^{47}$ Tumors in this clinical state continue to respond to secondary hormonal manipulations including ketoconazole, diethylstilbestrol, and abiraterone. ${ }^{48}$ When tumors truly become castrate-resistant, patients are offered docetaxel-based cytotoxic chemotherapy, which results in significant palliation of symptoms but only a modest prolongation in survival. ${ }^{49,50}$

In order to identify which prostate cancers require treatment and to improve existing therapies for those that do, there is a real sense of urgency among physicians and scientists to improve our ability to predict which prostate cancers are most virulent. This will require refinement of existing predictive and prognostic tools that are commonly employed by physicians to manage prostate cancer patients among different disease states. In this way, treatment will be applied only to patients who need it, while avoiding unnecessary treatment-related morbidity for those who do not. A major translational research effort is presently underway to address this need through the identification of novel biomarkers for prostate cancer.

\section{Biomarkers for prostate cancer}

A biomarker is defined as a characteristic that is measured and evaluated as an indicator of normal biologic processes, pathogenic processes, or pharmacologic responses to a therapeutic intervention. ${ }^{51}$ Biomarkers can thus provide information about the biology and natural history of the patient's disease (diagnostic/prognostic biomarker), the probability a patient will respond to a particular therapy (predictive biomarker), and "target engagement" by the therapy and modulation of the tumor phenotype in a clinically favorable manner (pharmacodynamic biomarker). ${ }^{52}$ There are four principal promises of biomarker research. ${ }^{10}$ First, biomarkers will improve current standards for defining disease by adding molecular analyses to more traditional criteria relying on patient symptoms and tissue pathology. Second, the clinical trials process will improve because biomarkers will provide "short-term" surrogate endpoints that substitute for traditional "long-term" endpoints such as time to disease recurrence and/or mortality from disease. Third, clinical care will improve based on the concept of personalized medicine - the idea that biomarkers will help physicians select therapies with the highest likelihood of success based on a patient's unique host and tumor biology. Fourth, the drug discovery process will become more efficient (and thereby less expensive) because biomarkers will help identify the most promising candidate therapeutics worthy of further development.

The most widely used biomarker in prostate cancer diagnosis and management is serum PSA., ${ }^{4,53}$ PSA is an androgen-regulated, serine protease encoded as a member of the tissue kallikrein family located on chromosome 19q13.4. Its normal physiologic function is to liquefy seminal fluid. PSA is synthesized in normal prostate tissue, benign prostatic hypertrophy, and in prostate cancer. The increase in serum PSA observed in prostate cancer patients is not due to increased expression of PSA on a cellular level per se, but rather tumor-associated disruption of normal prostate tissue architecture that leads to increased release of PSA into peripheral blood. ${ }^{54}$ PSA was first approved in 1986 by the Food and Drug Adminstration (FDA) to monitor response in patients treated for prostate cancer and subsequently as a diagnostic marker in 1994. Even at the time of its discovery, however, three inherent limitations of PSA as a biomarker were evident. First, PSA is not a prostate cancer specific antigen. Second, PSA does not reliably predict grade or stage of prostate cancer at diagnosis. Third, PSA reflects tumor volume but does not functionally contribute to the pathophysiology of tumor progression. Thus by itself, PSA does not distinguish clinically significant from insignificant prostate cancer. For these reasons, PSA is most useful as a predictive/prognostic tool when combined with other clinicopathologic parameters.

For patients with newly diagnosed prostate cancer, a combination of pretreatment PSA, Gleason score, and clinical stage have been incorporated into models to predict outcomes following radical prostatectomy or radiation therapy. Investigators at Johns Hopkins first used these three variables to predict the rate of finding disease that is not confined to the prostate at the time of surgery (by pathologic analysis of the surgical specimen). ${ }^{55,56}$ Since the decision to offer local therapy with curative intent depends on predicting whether the 
tumor is organ-confined versus nonorgan-confined, the now famous "Partin tables" have profoundly influenced clinical practice. Of course, not all patients with pathologically organconfined disease relapse, and not all patients with pathologically organ-confined cancers are cured. Thus the importance of predicting surgical outcome using pre-surgical covariates was and remains uncertain.

Additional models have been developed to predict outcomes following radical prostatectomy or radiation therapy. Based on the work of D'Amico, a combination of pre-therapy PSA, Gleason score, and clinical stage can be used to stratify patients into low (T1-T2a, Gleason score 2-6, and PSA $<10 \mathrm{ng} / \mathrm{mL}$ ), intermediate (T2b-T2c, Gleason score 7 , or PSA 10-20 ng/mL), high (T3a or Gleason score $8-10$ or PSA $>20 \mathrm{ng} / \mathrm{mL}$ ), and locally advanced (T3b-T4) groups that predict risk for both biochemical recurrence and survival following definitive local therapy (radical prostatectomy or radiation). ${ }^{34,57,58}$ Similarly, Kattan et al have developed postoperative nomograms for predicting prostate cancer recurrence after radical prostatectomy. ${ }^{59,60}$ These tools not only help guide recommendations for individual patients, but also help stratify patients for clinical trials. For example, low-risk patients can be directed towards "active surveillance" trials, while high-risk patients can be direct towards adjuvant/neoadjuvant trials.

Despite the efforts detailed above, tumors with identical PSA and clinicopathologic characteristics often display biologic and clinical heterogeneity. For example, some low-risk tumors rapidly progress while some high-risk tumors are relatively indolent. Accurate stratification is particularly challenging for Gleason 7 tumors, the most commonly reported score. Gleason 7 cancers (ie, $3+4$ or $4+3$ ) represent a clinically heterogeneous group with variable biologic potential and clinical outcomes. ${ }^{61}$ Despite efforts to improve stratification of Gleason 7 tumors using PSA and clinical stage, it is clear the Gleason system is inherently limited by the ability of light microscopic methodology to distinguish tumors with different biologic potential.

Investigational approaches to improve risk stratification of localized disease include assessing suspicious nodes or smallvolume extracapsular extension by magnetic resonance imaging (MRI) or positron emission tomography (PET), staging biopsies of seminal vesicles and extraprostatic tissue, and incorporation of molecular signatures derived from analysis of biopsy and/or prostatectomy specimens. For example, loss of tumor suppressor pathways (eg, p53) and gain of oncogene/ antiapoptotic pathways (eg, Bcl-2) contribute to prostate cancer progression. ${ }^{62,63}$ In addition to these and other "epithelial" events, the importance of the host-epithelial interaction in prostate cancer progression has been supported by evidence that pathways involved in paracrine regulation of normal stromal-epithelial interactions have also been implicated in prostate cancer progression. ${ }^{61,64,65}$ For example, sonic hedgehog and Src kinase signaling pathways are involved in normal bone development but their aberrant activation contributes to tumor progression. These pathways are currently being studied for biomarker development.

For patients with PSA-only recurrence after local therapy, PSA is commonly used as both a prognostic and predictive biomarker. ${ }^{39,66}$ The critical variable influencing therapy options for these patients is determining if the rise in PSA represents a local recurrence and/or microscopic metatastic disease. If patients are judged to have a local recurrence, salvage options are available, including radiation therapy after initial prostatectomy or cryosurgery after initial radiation therapy. For patients deemed to have micrometastatic disease, hormone ablative therapy is preferred. Both a rapid PSA velocity (rate of rise) and short PSA doubling time (PSADT) have been proposed as biomarkers to predict local versus distant metastases, and PSADT has additionally been predictive of risk for metastatic progression and cancer-specific mortality ${ }^{67-70}$ For patients who receive androgen ablation for PSA-only recurrent disease following local therapy with curative intent (surgery or radiation), PSA nadir is significantly associated with prostate cancer-specific mortality. ${ }^{71}$ At present, however, neither PSA velocity nor PSDAT have been prospectively validated, and there are still no established methods to accurately distinguish between local versus distant recurrence or to predict risk of metastatic progression and death in this patient group.

For patients with androgen-dependent metastatic disease, PSA nadir after androgen ablation is a strong predictor of overall survival, as is evidence for PSA progression after the nadir is achieved. ${ }^{72,73}$ Similarly, for patients with castrate resistant metastatic disease, the degree of PSA decline (eg, $\geqslant 50 \%$ ) achieved with chemotherapy predicts overall survival benefit. ${ }^{74,75}$ Because PSA is an androgen-regulated gene, PSA decline may be a better biomarker for response to hormonal ablative therapies rather than cytotoxic chemotherapies. This is one of the reasons circulating tumor cells may prove superior to PSA as a biomarker in patients receiving cytotoxic therapy for castrate-resistant disease. $^{5}$

Based on the above considerations, it is clear that novel biomarkers are needed in prostate cancer. PSA, considered alone or in combination with other clinicopathologic markers, 
has limitations as a biomarker and is not a valid surrogate for long-term benefit (ie, overall survival) in clinical trials testing novel therapies. Novel biomarkers will improve our ability to: distinguish clinically significant from insignificant cancers at diagnosis; predict which clinically significant tumors are at highest risk for relapse after local therapy so that adjuvant (or neoadjuvant) therapy can be applied; predict which patients with PSA-only relapse warrant aggressive therapy; predict response to therapies in the androgendependent and castrate-resistant disease states; and predict overall survival. Furthermore, in order to become useful in clinical practice, this biomarker should ideally be sensitive, specific, and reproducible.

\section{Caveolin-I in prostate cancer}

Cav-1 is a major structural coat protein of caveolae, specialized plasma membrane invaginations involved in multiple cellular functions including molecular transport, cell adhesion, and signal transduction. ${ }^{76,77}$ Caveolae are normally expressed in highly differentiated cells including epithelial cells, endothelia, cardiomyocytes, adipocytes, and osteoblasts. Caveolae have a specialized structure and function that distinguish them from general plasma membranes, lipid rafts, or clathrin-coated pits. ${ }^{78}$ In particular, caveolae contain high concentrations of signaling molecules including G-proteins, receptor tyrosine kinases, non-receptor tyrosine kinases, and endothelial nitric oxide synthase (eNOS).$^{79}$ These molecules interact with caveolae via protein-protein interactions with a 20-amino acid region of Cav-1 called the "caveolin-1 scaffolding domain" (CSD). ${ }^{80}$ Through CSD-mediated activities, caveolae act as compartments to organize signaling events from the cell surface to the inside of the cell and between specific intracellular organelles. ${ }^{81}$

Cav-1 was first implicated in cancer development when it was discovered to be a substrate for v-SRC in Rous sarcoma virus-transformed chicken embryo fibroblasts. ${ }^{82}$ Subsequent studies using both mouse and human cells revealed a complex role for Cav-1 in malignant transformation that is dependent on cell type, cell context, and growth conditions. For example, initial "gain of function" studies of Cav-1 ectopically expressed in breast cancer cells grown in vitro suggested that Cav-1 has tumor suppressor functions. ${ }^{83}$ Similar in vitro studies also suggested a tumor suppressor role for Cav-1 in colon cancer and sarcoma cell lines ${ }^{84} \mathrm{Cav}-1$ expression is also reduced in primary human breast cancers, colon cancers, and sarcomas, consistent with the notion that Cav-1 functions as a tumor suppressor in these tumor types. ${ }^{84}$
In contrast, immunohistochemistry analyses of primary human tumors of bladder, esophageal, breast, and prostate origin demonstrated aberrant overexpression of Cav-1 relative to normal tissues, suggesting an oncogenic role for Cav-1 for select tumor types. ${ }^{85-88}$ Importantly, immunohistochemical analysis of radical prostatectomy specimens obtained from patients with clinically localized tumors demonstrated that Cav-1 expression is positively associated with increasing Gleason grade, increasing Gleason score, lymph node involvement, and positive surgical margins. ${ }^{88}$ In a subset of lymph node-negative patients, multivariate analysis indicated that positive Cav-1 expression is an independent prognostic factor for a higher Gleason score $(>7)$, extraprostatic extension, seminal vesicle involvement, positive surgical margins, and shorter time to disease progression. In another study, patients with increased Cav-1 expression were at increased risk for developing an aggressive recurrence after surgery as defined by a PSADT of $<10$ months, failure to respond to salvage radiotherapy, and/or radiographically detected metastases. ${ }^{89}$ Taken together, these studies suggest that Cav-1 expression predicts development of prostate cancer with lethal potential.

In further support of this hypothesis, it is notable that Cav-1 was initially identified as a gene that is specifically upregulated in metastatic versus primary cancer cells in a mouse model system. ${ }^{87}$ Gain of function studies demonstrated that ectopically overexpressed Cav-1 protects prostate cancer cells from apoptotic stimuli..$^{90}$ Loss of function studies demonstrated that Cav-1 antisense cDNA converts castrate-resistant mouse prostate cancer cells to an androgendependent phenotype that is less prone to form metastases in vivo. ${ }^{91,92}$ As a corollary finding, selection for castrateresistant clones in vivo is associated with increased Cav-1 levels. These studies demonstrate that Cav-1 independently promotes prostate cancer cell survival, clonal expansion, castrate-resistance, and metastatic activities.

The frequency of Cav-1 positive cancers also underscores its distinct functional role in malignant progression. ${ }^{87}$ In normal prostate tissues, Cav-1 is abundantly expressed in stromal smooth muscle and endothelial cells but minimally expressed in both ductal and acinar epithelium. In contrast, in clinically localized prostate cancer (T1/T2aN0), Cav-1 is focally expressed by malignant epithelial cells in $\sim 14 \%$ of cases. Cav-1 expression proportionally increases in high-grade primary tumors with lymph node metastases (T3N1; 30\%) and in metastatic lymph nodes ( $56 \%)$. Increased Cav-1 expression also correlates with hormone ablative therapy. ${ }^{93}$ Analysis of primary tumor and metastatic specimens from 
patients with metastatic disease demonstrated that aberrant expression of Cav-1 was increased after hormone ablation in both primary (73\% versus $38 \%)$ and metastatic ( $82 \%$ versus $62 \%$ ) sites. The focal expression of Cav-1 in primary prostate cancers, the increase in Cav-1 expression in untreated metastases, and the additional increase in Cav-1 expression in tumors treated with hormone ablative therapy support the hypothesis that Cav-1 functions in progression-related events rather than local tumor growth.

The mechanism(s) for increased expression of Cav-1 in aggressive prostate cancer remains unclear. Cav-1 is regulated by testosterone, but at relatively modest levels when compared with PSA. ${ }^{92}$ This is evidenced by the relative paucity of Cav-1 expression in normal prostate acinar epithelium compared with expression of PSA. Cav-1 is located on 7q31.1, a conserved fragile site that is frequently deleted and/or amplified in human cancers. ${ }^{94,95}$ In addition, the gene promoter for Cav-1 possesses a "CpG" island that has been reported to be hypermethylated, an event generally associated with transcriptional repression. ${ }^{96}$ However, Cav-1 expression levels do not consistently correlate with genetic and/or methylation changes of the Cav-1 gene. ${ }^{97}$ More recently, an alternative epigenetic mechanism for increased Cav-1 expression has been proposed through the aberrant, cancer-specific loss of miR-205, a noncoding microRNA that normally silences Cav-1 expression. ${ }^{98}$

To further explore the significance of Cav-1 overexpression in human cancer, numerous preclinical studies have been performed to elucidate the precise molecular mechanism(s) for Cav-1 mediated oncogenic activity. Caveolae modulate diverse intracellular signaling pathways involved in regulation of cellular proliferation, apoptosis, endocytosis, and cholesterol trafficking. ${ }^{12}$ This is principally achieved by the interaction of Cav-1 with a large number of molecules in either a CSD-dependent or CSD-independent manner. Notably, Cav-1 modulates the function of many classic oncogenes, including v-Src, H-ras, and C-neu. ${ }^{84}$ Cav-1 inhibits or activates these pathways depending on the cell type and context.

In prostate cancer, Cav-1 interacts with and inhibits the serine/threonine protein phosphatases PP1 and PP2A in a CSD-dependent manner. ${ }^{90} \mathrm{PP} 1$ and PP2A are two major classes of phosphatases that act as tumor suppressors by maintaining Akt in a dephosphorylated, inactive state. Cav-1-mediated inhibition of PP1 and PP2A results in constitutive activation of prosurvival Akt-signaling. This has important implications in understanding the biology of clinically significant prostate cancer, since activation of Akt contributes to the development of aggressive, castrate-resistant disease. ${ }^{99}$ In this manner, overexpression of Cav-1 represents an alternative to PTEN loss in activating Akt in prostate cancer. Inactivating mutations of PTEN, another major regulator of the (PI3-K)/Akt pathway, are also common in advanced prostate cancers. ${ }^{100}$

More recently, Cav-1 has been shown to participate in a reciprocal, positive feedback loop with multiple growth factors implicated in prostate cancer progression. ${ }^{101}$ Cav-1 expression is increased in response to stimulation of prostate cancer cells by vascular endothelial growth factor (VEGF), platelet-derived growth factor (PDGF), fibroblast growth factor (FGF), and transforming growth factor $\beta 1$ (TGF- $\beta 1$ ). In turn, ectopic expression of Cav-1 increases expression of VEGF, TGF-1 $\beta$, and FGF2 protein levels through stabilization of mRNA transcripts for these genes in an Akt-dependent manner. Functionally, these events increase the invasive properties of prostate cancer cell lines in vitro, a phenotype associated with enhanced metastatic potential in vivo. ${ }^{101}$ Additional studies showed that Cav-1 stimulates VEGF-mediated autophoshorylation of VEGF receptor 2 (VEGFR2) in both human prostate cancer cells and human umbilical vein endothelial cells, illustrating the potential for Cav-1 to modulate angiogenesis through the cancer cell-host interaction. ${ }^{102}$

A major development in understanding Cav-1 biology was the discovery that Cav-1 is secreted by mouse and human prostate cancer cell lines. ${ }^{93}$ Phosphorylation of serine 80 in the CSD converts Cav-1 from an integral membrane protein to a secreted protein product. ${ }^{103} \mathrm{Cav}-1$ is then incorporated into other membrane-derived microvesicles that are secreted by prostate cancer cells, including "prostasomes" and “oncosomes". ${ }^{104,105}$ Importantly, secreted Cav-1 confers growth-promoting and anti-apoptotic properties to cell lines lacking endogenous Cav-1.93 This effect is mediated through uptake of Cav-1 by the Cav-1-negative prostate cancer epithelial cells. ${ }^{14}$ In a preclinical orthotopic mouse model of prostate cancer, Cav-1 antibody suppressed growth and metastases of a Cav-1 secreting, castrate-resistant cancer cell line. ${ }^{93}$ These data indicate that secreted Cav-1 is a biologically active autocrine-paracrine factor that contributes to malignant progression and further suggest that Cav-1 is a valid therapy target.

In addition to its effects on prostate cancer epithelial cells, secreted Cav-1 also modulates the microenvironment in a manner that promotes tumor growth through neoangiogenesis. Comparison of wild-type and Cav-1 knockout mice show that Cav-1 (-/-) mice display an impaired angiogenic response to exogenous stimuli, including basic fibroblast growth 
factor (bFGF) and a murine melanoma cell line (B16F10). ${ }^{106}$ Endothelial cells derived from Cav-1 knockout mice will internalize exogenous recombinant Cav-1 using lipid raft/ caveolae and clathrin-dependent endocytic pathways. ${ }^{14}$ Internalization of exogenous Cav-1 by Cav-1 (-/-) endothelial cells significantly increases their in vitro proangiogenic activities including migration, nitrous oxide production, and tubule formation. In vivo, orthotopic implantation of Cav-1secreting mouse prostate cancer cells into mice demonstrated that tumors are both larger and more vascular in Cav-1 $(+/+)$ versus Cav-1 (-/-) recipient hosts, although tumor-associated endothelial cells in Cav-1 (-/-) mice demonstrated uptake of tumor-derived Cav-1. ${ }^{14}$ In a second model system using a human prostate cancer cell line with inducible Cav-1 expression, Cav-1 secreting tumors were both larger and more vascular than control tumors in nude mice. ${ }^{14}$

Correlative studies in primary human prostate cancers further substantiate a role for Cav-1 in promoting tumorassociated neoangiogenesis. Analysis of 56 radical prostatectomy specimens revealed that tumor microvessel densities (MVD) were significantly higher in Cav-1 positive than Cav-1 negative tumors (based on Cav-1 scoring of tumor epithelial cells). ${ }^{107}$ MVD is a measure of tumor angiogenesis and increased MVD is associated with higher pathologic stage and shorter time to disease progression in men undergoing radical prostatectomy for localized disease. ${ }^{108}$ Interestingly, the percentage of Cav-1 positive tumor-associated endothelial cells (TAECs) was also significantly increased in Cav-1 positive versus negative tumors. Quantitative analysis indicated that the percentage of microvessels containing VEGFR2-postive TAEC was higher in Cav-1 positive than Cav-1 negative tumors. Taken together, these positive correlations support the concept that Cav-1 promotes pathologic neoangiogenesis in human prostate cancer.

Initial studies have demonstrated that secreted Cav-1 is a novel biomarker for predicting clinically significant prostate cancer. Secreted Cav-1 can be measured in peripheral blood using an ELISA that is sensitive, specific, and reproducible. ${ }^{13}$ The median serum Cav-1 level is significantly higher in patients with clinically localized prostate cancer than in men with benign prostatic hypertrophy or healthy controls. ${ }^{13}$ In a group of 419 patients undergoing radical prostatectomy for localized disease, patients with high preoperative serum Cav-1 levels had a 2.7-fold greater risk of experiencing a biochemical recurrence compared with patients with low values. ${ }^{109}$ In a subset analysis of patients with high-risk disease (PSA $>10$ ), those with high preoperative serum Cav-1 levels had a 2.4-fold greater risk of developing biochemical recurrence compared with those with low Cav-1 levels. Importantly, when both Cav-1 and biopsy Gleason score were combined into a multivariate Cox proportional hazard model, the analysis of both variables more accurately predicted for risk of biochemical recurrence than either variable alone. Lastly, Cav-1 could stratify patients with Gleason 7 tumors into high- and low-risk recurrence groups after adjusting for PSA levels. ${ }^{109}$

These data suggest that measurements of tissue Cav-1 and serum Cav-1 expression levels represent clinically useful prognostic and predictive biomarkers for prostate cancer (see Table 1). In patients with localized disease at diagnosis, Cav-1 improves the ability of current predictive tools to distinguish patients with clinically significant disease from those with insignificant disease. In this way, serum and tissue Cav-1 levels supplement (rather than supplant) other established predictive variables including serum PSA, Gleason score, and clinical stage. Future research efforts will test whether Cav-1 expression levels identify patients with localized disease who would benefit from neoadjuvant and/or adjuvant therapy.

Notably, when compared with serum PSA, serum Cav-1 more specifically identifies clinically aggressive disease. This likely reflects the fact that, unlike PSA, Cav-1 biologically contributes to malignant progression and the development of castrate-resistance. For the same reason, serum Cav-1 measurement may also prove useful as a biomarker in other prostate cancer disease states (see Figure 1). For example,

Table I Rationale for developing caveolin-I as a biomarker for clinically significant prostate cancer

- Cav-I expression is significantly increased in prostate cancer versus normal epithelium.

- Cav-I expression is increased in metastatic versus localized prostate cancer.

- In contrast with PSA, Cav-I is an oncogene that significantly contributes to the biology of aggressive disease.

- Prostate cancer cells secrete Cav-I into the tumor microenvironment.

- Like endogenous Cav-I, secreted Cav-I promotes malignant progression through antiapoptotic activities, stimulation of angiogenesis, castrate-resistant growth, and metastases formation.

- Secreted Cav-I can be reliably and reproducibly measured from peripheral blood using a Cav-I-specific ELISA.

- Serum Cav-I levels are significantly higher in men with clinically localized prostate cancer than in men with BPH or healthy controls.

- High pretreatment levels of serum Cav-I predict for a shorter time to biochemical recurrence in patients undergoing radical prostatectomy for localized disease.

Abbreviations: BPH, benign prostatic hypertrophy; Cav-I, caveolin- I; ELISA, enzymelinked immunosorbent assay; PSA, prostate-specific antigen. 


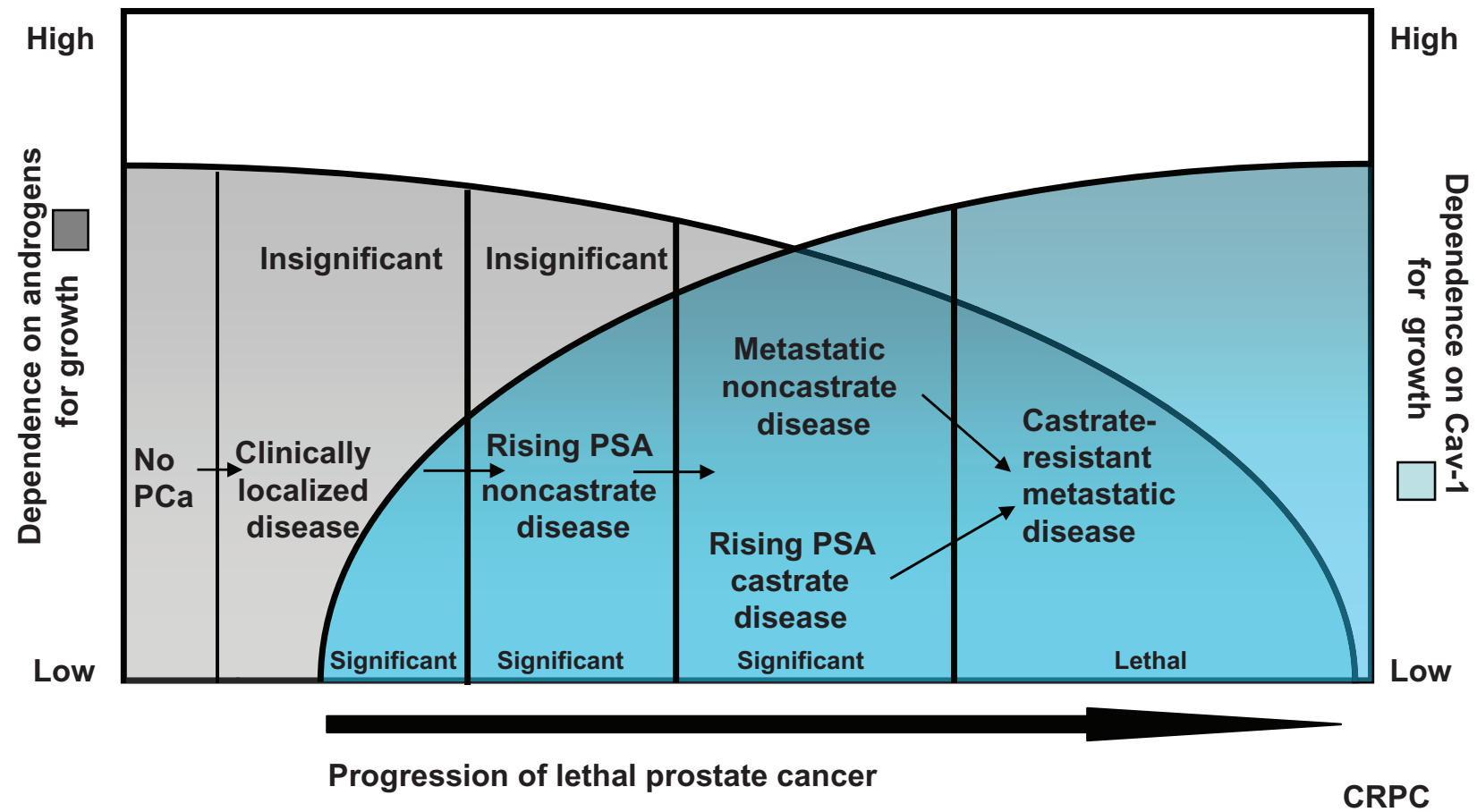

Figure I The clinical utility of Cav-I as a biomarker reflects its biologic contribution to malignant progression. Prostate cancer disease states reflecting the development of lethal disease (x-axis) are depicted in relation to dependence on androgens (left y-axis: from high to low) for growth and increased dependence on Cav-I for growth (right $y$-axis: from low to high). ${ }^{29}$ Malignant progression is associated with reduced dependence on androgens (from high to low) and increased dependence on Cav-I for growth (from low to high). Serum Cav-I is a useful biomarker to distinguish clinically significant from insignificant localized disease. It is also anticipated that serum Cav-I will be a clinically useful biomarker for other prostate cancer disease states. For example, Cav-I levels may help identify which patients with PSA-only recurrent disease require aggressive therapy, and which patients with castrate-resistant disease respond to systemic therapy.

Abbreviations: Cav-I, caveolin-I; PSA, prostate-specific antigen.

in patients with PSA-only recurrent disease following local therapy, analysis of serum Cav-1 levels may help determine which of these patients warrant aggressive therapy. Similarly, in patients with castrate-resistant metastatic disease, serum Cav-1 levels may be useful in monitoring response to therapy. Since Cav-1 critically modulates both the epithelial and host tissue microenvironment, the success of novel treatment strategies that target both compartments should correlate with serum Cav-1 responses.

\section{Summary}

A growing body of preclinical and clinical data has established the importance of Cav-1 in the development of virulent prostate cancer. As an oncogene, Cav-1 protects prostate cancer epithelial cells from apoptotic stimuli, enhances prosurvival pathways mediated by multiple growth factors involved in prostate cancer progression, and supports castrate-resistant growth. Cav-1 also contributes to the metastatic potential of prostate cancer through induction of tumor-associated neoangiogenesis in the host microenvironment. Because it is a secreted protein that can be reliably measured in peripheral blood by ELISA, Cav-1 has enormous potential as a novel biomarker in prostate cancer.
Preliminary studies suggest that serum Cav-1 enhances the performance of existing tools that rely on serum PSA, Gleason score, and clinical stage to predict outcomes for patients who receive treatment for localized disease. Serum Cav-1 should also prove useful as a prognostic/predictive biomarker for patients with PSA-only recurrent disease and in patients receiving therapy for metastatic disease. Additional studies are currently underway to validate this promising biomarker.

\section{Disclosure}

The authors report no conflicts of interest in this work.

\section{References}

1. Cher ML, Towler DA, Rafii S, et al. Cancer interaction with the bone microenvironment: A workshop of the National Institutes of Health Tumor Microenvironment Study Section. Am J Pathol. 2006;168: 1405-1412.

2. Logothetis CJ, Lin SH. Osteoblasts in prostate cancer metastasis to bone. Nat Rev Cancer. 2005;5:21-28.

3. Rao AR, Motiwala HG, Karim OM. The discovery of prostate-specific antigen. BJU Int. 2008;101:5-10.

4. Lilja H, Ulmert D, Vickers AJ. Prostate-specific antigen and prostate cancer: Prediction, detection and monitoring. Nat Rev Cancer. 2008;8:268-278. 
5. de Bono JS, Scher HI, Montgomery RB, et al. Circulating tumor cells predict survival benefit from treatment in metastatic castration-resistant prostate cancer. Clin Cancer Res. 2008;14:6302-6309.

6. Schiffer E. Biomarkers for prostate cancer. World J Urol. 2007;25: $557-562$.

7. Reynolds MA. Molecular alterations in prostate cancer. Cancer Lett. 2008;271:13-24.

8. Masters JR. Clinical applications of expression profiling and proteomics in prostate cancer. Anticancer Res. 2007;27:1273-1276.

9. Liszewski K. Optimizing Utilization of Biomarkers. Genetic Engineering and Biotechnology News. 28(12): Jun 15, 2009.

10. Corn PG. Methylation marks the path for biomarker development in breast cancer research. Cancer Biol Ther. 2009;8(23):2260-2262.

11. Thompson TC, Tahir SA, Li L, et al. The role of caveolin-1 in prostate cancer: Clinical implications. Prostate Cancer Prostatic Dis. 2010;13(1):6-11.

12. Carver LA, Schnitzer JE. Caveolae: Mining little caves for new cancer targets. Nat Rev Cancer. 2003;3:571-581.

13. Tahir SA, Ren C, Timme TL, et al. Development of an immunoassay for serum caveolin-1: A novel biomarker for prostate cancer. Clin Cancer Res. 2003;9:3653-3659.

14. Tahir SA, Yang G, Goltsov AA, et al. Tumor cell-secreted caveolin-1 has proangiogenic activities in prostate cancer. Cancer Res. 2008;68: 731-739.

15. Jemal A, Siegel R, Ward E, Hao Y, Xu J, Thun MJ. Cancer statistics, 2009. CA Cancer J Clin. 2009;59:225-249.

16. Gann PH. Risk factors for prostate cancer. Rev Urol. 2002;4 Suppl 5: S3-S10.

17. Patel AR, Klein EA. Risk factors for prostate cancer. Nat Clin Pract Urol. 2009;6:87-95.

18. Heron M, Hoyert DL, Murphy SL, Xu J, Kochanek KD, Tejada-Vera B. Deaths: Final data for 2006. Natl Vital Stat Rep. 2009;57:1-134.

19. Calle EE, Rodriguez C, Walker-Thurmond K, Thun MJ. Overweight, obesity, and mortality from cancer in a prospectively studied cohort of U.S. adults. $N$ Engl J Med. 2003;348:1625-1638.

20. Strom SS, Wang X, Pettaway CA, et al. Obesity, weight gain, and risk of biochemical failure among prostate cancer patients following prostatectomy. Clin Cancer Res. 2005;11:6889-6894.

21. Xu J, Gillanders EM, Isaacs SD, et al. Genome-wide scan for prostate cancer susceptibility genes in the Johns Hopkins hereditary prostate cancer families. Prostate. 2003;57:320-325.

22. Gayther SA, de Foy KA, Harrington P, et al. The frequency of germline mutations in the breast cancer predisposition genes BRCA1 and BRCA2 in familial prostate cancer. The Cancer Research Campaign/ British Prostate Group United Kingdom Familial Prostate Cancer Study Collaborators. Cancer Res. 2000;60:4513-4518.

23. Adolfsson J. Commentary: On the incidence of histological prostate cancer and the probable diagnosis of cases with tumours too small to produce symptoms or to attract attention on physical examination - the findings of Dr. Arnold Rice Rich. Int J Epidemiol. 2007;36:285-287.

24. Winkler MH, Livni N, Mannion EM, Hrouda D, Christmas T. Characteristics of incidental prostatic adenocarcinoma in contemporary radical cystoprostatectomy specimens. BJU Int. 2007;99:554-558.

25. Johansson JE, Holmberg L, Johansson S, Bergstrom R, Adami HO. Fifteen-year survival in prostate cancer. A prospective, population-based study in Sweden. JAMA. 1997;277:467-471.

26. Johansson JE, Andren O, Andersson SO, et al. Natural history of early, localized prostate cancer. JAMA. 2004;291:2713-2719.

27. Bastian PJ, Carter BH, Bjartell A, et al. Insignificant prostate cancer and active surveillance: From definition to clinical implications. Eur Urol. 2009;55:1321-1330.

28. Saitoh H, Hida M, Shimbo T, Nakamura K, Yamagata J, Satoh T. Metastatic patterns of prostatic cancer. Correlation between sites and number of organs involved. Cancer. 1984;54:3078-3084.

29. Scher HI, Heller G. Clinical states in prostate cancer: Toward a dynamic model of disease progression. Urology. 2000;55:323-327.
30. Scher HI, Halabi S, Tannock I, et al. Design and end points of clinical trials for patients with progressive prostate cancer and castrate levels of testosterone: Recommendations of the Prostate Cancer Clinical Trials Working Group. J Clin Oncol. 2008;26:1148-1159.

31. Catalona WJ, Smith DS, Ratliff TL, Basler JW. Detection of organ-confined prostate cancer is increased through prostate-specific antigen-based screening. JAMA. 1993;270:948-954.

32. Lu-Yao GL, Friedman M, Yao SL. Use of radical prostatectomy among Medicare beneficiaries before and after the introduction of prostate specific antigen testing. J Urol. 1997;157:2219-2222.

33. D'Amico AV, Whittington R, Malkowicz SB, et al. Biochemical outcome after radical prostatectomy, external beam radiation therapy, or interstitial radiation therapy for clinically localized prostate cancer. JAMA. 1998;280:969-974.

34. D'Amico AV, Moul J, Carroll PR, Sun L, Lubeck D, Chen MH. Cancer-specific mortality after surgery or radiation for patients with clinically localized prostate cancer managed during the prostate-specific antigen era. J Clin Oncol. 2003;21:2163-2172.

35. Bill-Axelson A, Holmberg L, Ruutu M, et al. Radical prostatectomy versus watchful waiting in early prostate cancer. $N$ Engl $J$ Med. 2005;352:1977-1984.

36. Andriole GL, Crawford ED, Grubb RL III, et al. Mortality results from a randomized prostate-cancer screening trial. $N$ Engl $J$ Med. 2009;360:1310-1319.

37. Schroder FH, Hugosson J, Roobol MJ, et al. Screening and prostate-cancer mortality in a randomized European study. $N$ Engl J Med. 2009;360:1320-1328.

38. Autier P, Boniol M, Hery C, Masuyer E, Ferlay J. Cancer survival statistics should be viewed with caution. Lancet Oncol. 2007;8:1050-1052.

39. Aranha O, Vaishampayan U. PSA relapse prostate cancer: The importance of tailored therapy. Urol Oncol. 2004;22:62-69.

40. Thompson IM, Tangen CM, Paradelo J, et al. Adjuvant radiotherapy for pathological T3N0M0 prostate cancer significantly reduces risk of metastases and improves survival: Long-term followup of a randomized clinical trial. J Urol. 2009;181:956-962.

41. Messing EM, Manola J, Sarosdy M, Wilding G, Crawford ED, Trump D. Immediate hormonal therapy compared with observation after radical prostatectomy and pelvic lymphadenectomy in men with node-positive prostate cancer. N Engl J Med. 1999;341:1781-1788.

42. Pilepich MV, Winter K, Lawton CA, et al. Androgen suppression adjuvant to definitive radiotherapy in prostate carcinoma - longterm results of phase III RTOG 85-31. Int J Radiat Oncol Biol Phys. 2005;61:1285-1290.

43. Pound CR, Partin AW, Eisenberger MA, Chan DW, Pearson JD, Walsh PC. Natural history of progression after PSA elevation following radical prostatectomy. JAMA. 1999;281:1591-1597.

44. Bhandari MS, Crook J, Hussain M. Should intermittent androgen deprivation be used in routine clinical practice? J Clin Oncol. 2005;23:8212-8218.

45. Crawford ED. Hormonal therapy in prostate cancer: Historical approaches. Rev Urol. 2004;6 Supp1 7:S3-S11.

46. Feldman BJ, Feldman D. The development of androgen-independent prostate cancer. Nat Rev Cancer. 2001;1:34-45.

47. Scher HI, Sawyers CL. Biology of progressive, castration-resistant prostate cancer: Directed therapies targeting the androgen-receptor signaling axis. J Clin Oncol. 2005;23:8253-8261.

48. Van Allen EM, Ryan CJ. Novel secondary hormonal therapy in advanced prostate cancer: An update. Curr Opin Urol. 2009;19:315-321.

49. Tannock IF, de WR, Berry WR, et al. Docetaxel plus prednisone or mitoxantrone plus prednisone for advanced prostate cancer. $N$ Engl JMed. 2004;351:1502-1512.

50. Petrylak DP, Tangen CM, Hussain MH, et al. Docetaxel and estramustine compared with mitoxantrone and prednisone for advanced refractory prostate cancer. $N$ Engl J Med. 2004;351:1513-1520.

51. Manne U, Srivastava RG, Srivastava S. Recent advances in biomarkers for cancer diagnosis and treatment. Drug Discov Today. 2005;10:965-976. 
52. Sawyers CL. The cancer biomarker problem. Nature. 2008;452: 548-552.

53. Balk SP, Ko YJ, Bubley GJ. Biology of prostate-specific antigen. J Clin Oncol. 2003;21:383-391.

54. Stamey TA, Yang N, Hay AR, McNeal JE, Freiha FS, Redwine E. Prostate-specific antigen as a serum marker for adenocarcinoma of the prostate. N Engl J Med. 1987;317:909-916.

55. Partin AW, Kattan MW, Subong EN, et al. Combination of prostatespecific antigen, clinical stage, and Gleason score to predict pathological stage of localized prostate cancer. A multi-institutional update. JAMA. 1997;277:1445-1451.

56. Makarov DV, Trock BJ, Humphreys EB, et al. Updated nomogram to predict pathologic stage of prostate cancer given prostate-specific antigen level, clinical stage, and biopsy Gleason score (Partin tables) based on cases from 2000 to 2005. Urology. 2007;69:1095-1101.

57. D'Amico AV, Whittington R, Malkowicz SB, et al. Biochemical outcome after radical prostatectomy, external beam radiation therapy, or interstitial radiation therapy for clinically localized prostate cancer JAMA. 1998;280:969-974.

58. Hernandez DJ, Nielsen ME, Han M, Partin AW. Contemporary evaluation of the D'amico risk classification of prostate cancer. Urology. 2007;70:931-935.

59. Kattan MW, Wheeler TM, Scardino PT. Postoperative nomogram for disease recurrence after radical prostatectomy for prostate cancer. J Clin Oncol. 1999;17:1499-1507.

60. Graefen M, Karakiewicz PI, Cagiannos I, et al. Validation study of the accuracy of a postoperative nomogram for recurrence after radical prostatectomy for localized prostate cancer. J Clin Oncol. 2002;20 951-956.

61. Stark JR, Perner S, Stampfer MJ, et al. Gleason score and lethal prostate cancer: Does 3 + 4=4 + 3? J Clin Oncol. 2009;27:3459-3464.

62. Navone NM, Labate ME, Troncoso P, et al. p53 mutations in prostate cancer bone metastases suggest that selected $\mathrm{p} 53$ mutants in the primary site define foci with metastatic potential. J Urol. 1999;161:304-308.

63. McDonnell TJ, Navone NM, Troncoso P, et al. Expression of bcl-2 oncoprotein and p53 protein accumulation in bone marrow metastases of androgen independent prostate cancer. J Urol. 1997;157: 569-574.

64. Sanchez P, Hernandez AM, Stecca B, et al. Inhibition of prostate cancer proliferation by interference with SONIC HEDGEHOG-GLI1 signaling. Proc Natl Acad Sci U S A. 2004;101:12561-12566.

65. Zunich SM, Douglas T, Valdovinos M, et al. Paracrine sonic hedgehog signalling by prostate cancer cells induces osteoblast differentiation. Mol Cancer. 2009;8:12.

66. Pound CR, Brawer MK, Partin AW. Evaluation and treatment of men with biochemical prostate-specific antigen recurrence following definitive therapy for clinically localized prostate cancer. Rev Urol. 2001;3:72-84.

67. Partin AW, Pearson JD, Landis PK, et al. Evaluation of serum prostate-specific antigen velocity after radical prostatectomy to distinguish local recurrence from distant metastases. Urology. 1994;43:649-659

68. Patel A, Dorey F, Franklin J, deKernion JB. Recurrence patterns after radical retropubic prostatectomy: Clinical usefulness of prostate specific antigen doubling times and log slope prostate specific antigen. $J$ Urol. 1997;158:1441-1445.

69. Cannon GM Jr, Walsh PC, Partin AW, Pound CR. Prostate-specific antigen doubling time in the identification of patients at risk for progression after treatment and biochemical recurrence for prostate cancer. Urology. 2003;62 Suppl 1:2-8.

70. D'Amico AV, Moul JW, Carroll PR, Sun L, Lubeck D, Chen MH. Surrogate end point for prostate cancer-specific mortality after radical prostatectomy or radiation therapy. J Natl Cancer Inst. 2003;95:1376-1383.

71. Stewart AJ, Scher HI, Chen MH, et al. Prostate-specific antigen nadir and cancer-specific mortality following hormonal therapy for prostatespecific antigen failure. J Clin Oncol. 2005;23:6556-6560.
72. Hussain M, Tangen CM, Higano C, et al. Absolute prostate-specific antigen value after androgen deprivation is a strong independent predictor of survival in new metastatic prostate cancer: Data from Southwest Oncology Group Trial 9346 (INT-0162). J Clin Oncol. 2006;24:3984-3990.

73. Hussain M, Goldman B, Tangen C, et al. Prostate-specific antigen progression predicts overall survival in patients with metastatic prostate cancer: Data from Southwest Oncology Group Trials 9346 (Intergroup Study 0162) and 9916. J Clin Oncol. 2009;27:2450-2456.

74. Bubley GJ, Carducci M, Dahut W, et al. Eligibility and response guidelines for phase II clinical trials in androgen-independent prostate cancer: Recommendations from the Prostate-Specific Antigen Working Group. J Clin Oncol. 1999;17:3461-3467.

75. Petrylak DP, Ankerst DP, Jiang CS, et al. Evaluation of prostate-specific antigen declines for surrogacy in patients treated on SWOG 99-16. J Natl Cancer Inst. 2006;98:516-521.

76. Shaul PW, Anderson RG. Role of plasmalemmal caveolae in signal transduction. Am J Physiol. 1998;275:L843-L851.

77. Sternberg PW, Schmid SL. Caveolin, cholesterol and Ras signalling. Nat Cell Biol. 1999;1:E35-E37.

78. Anderson RG, Jacobson K. A role for lipid shells in targeting proteins to caveolae, rafts, and other lipid domains. Science. 2002;296: $1821-1825$

79. Carver LA, Schnitzer JE, Anderson RG, Mohla S. Role of caveolae and lipid rafts in cancer: Workshop summary and future needs. Cancer Res. 2003;63:6571-6574.

80. Li S, Couet J, Lisanti MP. Src tyrosine kinases, Galpha subunits, and $\mathrm{H}$-Ras share a common membrane-anchored scaffolding protein, caveolin. Caveolin binding negatively regulates the auto-activation of Src tyrosine kinases. J Biol Chem. 1996;271:29182-29190.

81. Shatz M, Liscovitch M. Caveolin-1: A tumor-promoting role in human cancer. Int J Radiat Biol. 2008;84:177-189.

82. Glenney JR Jr. Tyrosine phosphorylation of a $22-\mathrm{kDa}$ protein is correlated with transformation by Rous sarcoma virus. J Biol Chem. 1989;264:20163-20166.

83. Razani B, Schlegel A, Liu J, Lisanti MP. Caveolin-1, a putative tumour suppressor gene. Biochem Soc Trans. 2001;29:494-499.

84. Williams TM, Lisanti MP. Caveolin-1 in oncogenic transformation, cancer, and metastasis. Am J Physiol Cell Physiol. 2005;288: C494-C506.

85. Rajjayabun PH, Garg S, Durkan GC, Charlton R, Robinson MC, Mellon JK. Caveolin-1 expression is associated with high-grade bladder cancer. Urology. 2001;58:811-814.

86. Kato K, Hida Y, Miyamoto M, et al. Overexpression of caveolin-1 in esophageal squamous cell carcinoma correlates with lymph node metastasis and pathologic stage. Cancer. 2002;94:929-933.

87. Yang G, Truong LD, Timme TL, et al. Elevated expression of caveolin is associated with prostate and breast cancer. Clin Cancer Res. 1998;4:1873-1880.

88. Yang G, Truong LD, Wheeler TM, Thompson TC. Caveolin-1 expression in clinically confined human prostate cancer: A novel prognostic marker. Cancer Res. 1999;59:5719-5723.

89. Karam JA, Lotan Y, Roehrborn CG, Ashfaq R, Karakiewicz PI, Shariat SF. Caveolin-1 overexpression is associated with aggressive prostate cancer recurrence. Prostate. 2007;67:614-622.

90. Li L, Ren CH, Tahir SA, Ren C, Thompson TC. Caveolin-1 maintains activated Akt in prostate cancer cells through scaffolding domain binding site interactions with and inhibition of serine/threonine protein phosphatases PP1 and PP2A. Mol Cell Biol. 2003;23: 9389-9404.

91. Nasu Y, Timme TL, Yang G, et al. Suppression of caveolin expression induces androgen sensitivity in metastatic androgen-insensitive mouse prostate cancer cells. Nat Med. 1998;4:1062-1064.

92. Li L, Yang G, Ebara S, et al. Caveolin-1 mediates testosterone-stimulated survival/clonal growth and promotes metastatic activities in prostate cancer cells. Cancer Res. 2001;61:4386-4392. 
93. Tahir SA, Yang G, Ebara S, et al. Secreted caveolin-1 stimulates cell survival/clonal growth and contributes to metastasis in androgeninsensitive prostate cancer. Cancer Res. 2001;61:3882-3885.

94. Engelman JA, Zhang XL, Lisanti MP. Genes encoding human caveolin-1 and -2 are co-localized to the D7S522 locus (7q31.1), a known fragile site (FRA7G) that is frequently deleted in human cancers. FEBS Lett. 1998;436:403-410.

95. Nupponen NN, Kakkola L, Koivisto P, Visakorpi T. Genetic alterations in hormone-refractory recurrent prostate carcinomas. Am J Pathol. 1998;153:141-148.

96. Cui J, Rohr LR, Swanson G, Speights VO, Maxwell T, Brothman AR. Hypermethylation of the caveolin-1 gene promoter in prostate cancer. Prostate. 2001;46:249-256.

97. Bachmann N, Haeusler J, Luedeke M, et al. Expression changes of CAV1 and EZH2, located on 7q31 approximately q36, are rarely related to genomic alterations in primary prostate carcinoma. Cancer Genet Cytogenet. 2008;182:103-110.

98. Gandellini P, Folini M, Longoni N, et al. miR-205 Exerts tumorsuppressive functions in human prostate through down-regulation of protein kinase Cepsilon. Cancer Res. 2009;69:2287-2295.

99. Xin L, Teitell MA, Lawson DA, Kwon A, Mellinghoff IK, Witte ON. Progression of prostate cancer by synergy of AKT with genotropic and nongenotropic actions of the androgen receptor. Proc Natl Acad Sci U S A. 2006;103:7789-7794.

100. Cantley LC, Neel BG. New insights into tumor suppression: PTEN suppresses tumor formation by restraining the phosphoinositide 3-kinase/AKT pathway. Proc Natl Acad Sci U S A. 1999;96: 4240-4245.

101. Li L, Ren C, Yang G, Goltsov AA, Tabata K, Thompson TC. Caveolin-1 promotes autoregulatory, Akt-mediated induction of cancer-promoting growth factors in prostate cancer cells. Mol Cancer Res. 2009;7:1781-1791.
102. Tahir SA, Park S, Thompson TC. Caveolin-1 regulates VEGFstimulated angiogenic activities in prostate cancer and endothelial cells. Cancer Biol Ther. 2009;8.

103. Schlegel A, Arvan P, Lisanti MP. Caveolin-1 binding to endoplasmic reticulum membranes and entry into the regulated secretory pathway are regulated by serine phosphorylation. Protein sorting at the level of the endoplasmic reticulum. J Biol Chem. 2001;276: 4398-4408.

104. Llorente A, de Marco MC, Alonso MA. Caveolin-1 and MAL are located on prostasomes secreted by the prostate cancer PC-3 cell line. $J$ Cell Sci. 2004;117:5343-5351.

105. Lu Q, Zhang J, Allison R, et al. Identification of extracellular delta-catenin accumulation for prostate cancer detection. Prostate. 2009;69:411-418.

106. Woodman SE, Ashton AW, Schubert W, et al. Caveolin-1 knockout mice show an impaired angiogenic response to exogenous stimuli. Am J Pathol. 2003;162:2059-2068.

107. Yang G, Addai J, Wheeler TM, et al. Correlative evidence that prostate cancer cell-derived caveolin-1 mediates angiogenesis. Hum Pathol. 2007;38:1688-1695.

108. Bono AV, Celato N, Cova V, Salvadore M, Chinetti S, Novario R. Microvessel density in prostate carcinoma. Prostate Cancer Prostatic Dis. 2002;5:123-127.

109. Tahir SA, Frolov A, Hayes TG, et al. Preoperative serum caveolin-1 as a prognostic marker for recurrence in a radical prostatectomy cohort. Clin Cancer Res. 2006;12:4872-4875.
Cancer Management and Research

\section{Publish your work in this journal}

Cancer Management and Research is an international, peer-reviewed open access journal focusing on cancer research and the optimal use of preventative and integrated treatment interventions to achieve improved outcomes, enhanced survival and quality of life for the cancer patient. The journal welcomes original research, clinical \& epidemiological

\section{Dovepress}

studies, reviews \& evaluations, guidelines, expert opinion \& commentary, case reports \& extended reports. The manuscript management system is completely online and includes a very quick and fair peerreview system, which is all easy to use. Visit http://www.dovepress.com/ testimonials.php to read real quotes from published authors. 